\title{
Internet como herramienta didáctica en la formación académica en alumnos de nivel medio superior
} Internet as a teaching tool in academic formation in Higher Level Education students Internet como ferramenta de ensino na formação de estudantes académicos de topo de nível médio

DOI: http://dx.doi.org/10.23913/ricsh.v6i11.114

Gilberto Mejía-Salazar Universidad Autónoma de Nayarit, México Solkjaer88@hotmail.com

Ricardo Gómez-Álvarez Universidad Autónoma de Nayarit, México rgomeza_15@hotmail.com

\section{Resumen}

El siguiente trabajo de investigación se realizó con alumnos de nivel medio superior, asimismo, el objetivo principal es analizar la utilidad que se le da a internet como herramienta didáctica dentro del aprendizaje educativo de los alumnos. De esta manera, se aplicó un cuestionario estructurado con 10 preguntas a una muestra de 192 alumnos conformada por los grados de primero, segundo y tercer año de la preparatoria 14. Finalmente, la información adquirida se procesó en el programa estadístico SPSS versión 19, obteniendo los respectivos resultados. Por lo tanto, Internet ha dado inicio a desarrollar nuevos modelos y estrategias de aprendizaje, para fortalecer la formación de los estudiantes, así pues, el integrar estas herramientas da la oportunidad de ejercer habilidades autodidactas dentro de los campos educativos y seguir con una educación basada en Tecnologías de Información y Comunicación. 
Palabras clave: Búsqueda en línea, Educación, Internet, TIC.

\section{Abstract}

The following research work was carried out with Higher Level Education students, also, the main objective is to analyze the utility that is given to the internet as a teaching tool within the educational learning. This way, a structured questionnaire with 10 questions was applied to a sample of 192 students formed by the first, second and third degrees of the preparatory \#14. Finally, the information gained was processed in the statistical program SPSS version 19, obtaining the respective results. Therefore, Internet has given home to develop new models and learning strategies to strengthen the training of the students, thus integrating these tools gives the opportunity to practise self-taught skills in the educational fields and follow with an education based on Information and Communication Technologies (ICT).

Key words: online search, Education, Internet, Information and Communication Technology (ICT).

\section{Resumo}

A presente pesquisa foi realizada com alunos de nível médio, também, o objetivo principal é analisar a utilidade é dada Internet como uma ferramenta de ensino na aprendizagem dos alunos da educação. Assim, um questionário estruturado com 10 perguntas foi aplicado a uma amostra de 192 alunos fizeram-se os graus de primeiro, segundo e terceiro ano do ensino médio 14. Finalmente, as informações obtidas foram processados em SPSS versão 19, obtendo os respectivos resultados. Portanto, a Internet começou a desenvolver novos modelos e estratégias de aprendizagem para fortalecer a formação de estudantes, assim, a integração dessas ferramentas dá a oportunidade de exercitar habilidades autodidatas nos domínios educativo e continuar com uma educação baseada Tecnologia da informação e Comunicação. Palavras-chave: pesquisa on-line, Educação, Internet, TIC.

Fecha Recepción: Junio $2016 \quad$ Fecha Aceptación: Diciembre 2016 


\section{Introducción}

Internet es una poderosa herramienta para ayudar a la difusión del conocimiento y la educación, de hecho, es una de las mayores fuentes de información disponibles, se dice que estamos en la era de la comunicación y el conocimiento, de ahí la importancia de esta red de redes que actualmente se extiende por todas partes del mundo, reduciendo considerablemente el tiempo y esfuerzo empleado en la búsqueda del saber y la información (Pérez y Florido, 2003).

Por lo tanto, la red es un recurso formidable para enriquecer la perspectiva de nuestros alumnos y el proceso de analizar, valorar, integrar información diversa es la esencia del proceso de construcción de conocimientos (Adell, 2004). De lo anterior, en internet se puede encontrar información de todo tipo, desde la más útil hasta la más innecesaria, trivial y ofensiva. La forma en que podemos generar en nosotros mismos la capacidad de discriminar qué información es la que nos puede resultar útil y cual debemos descartar por inoperante es otra de las cuestiones que interesan a los autores (Dari, 2004).

Asimismo, el siguiente trabajo de investigación tiene como objetivo principal, analizar el uso del internet como herramienta didáctica en el proceso de aprendizaje educativo en los alumnos del nivel medio superior de la preparatoria 14 de la Universidad Autónoma de Nayarit. De esta manera, se plantea la siguiente hipótesis, el Internet es la herramienta didáctica más utilizada para el proceso de aprendizaje de los alumnos del nivel medio superior de la preparatoria 14 de la Universidad Autónoma de Nayarit.

Así pues, se aplicó un cuestionario estructurado con 10 preguntas a una muestra de 192 alumnos conformada por los grados de primero, segundo y tercer año de la preparatoria 14. Finalmente, la información adquirida se procesó en el programa estadístico SPSS versión 19, obteniendo los respectivos resultados. 


\section{Internet: herramienta de aprendizaje}

Así pues, Internet se ha convertido en el soporte técnico imprescindible para el desarrollo de nuevos modelos de enseñanza a la vez que es una potente herramienta didáctica que permite el acceso a una cantidad ingente de información y abre nuevos canales de comunicación, rompiendo, como se ha dicho tantas veces, barreras temporales y espaciales conceptual (López y Morcillo, 2007). Ciertamente existen cada vez más portales educativos en internet en los que podemos encontrar recursos didácticos para el aula, pero aún son insuficientes -sobre todo en español- y, en la mayoría de los casos, estos recursos constituyen documentos o actividades encaminadas a la búsqueda de información o para reforzar conocimientos dentro del ámbito conceptual (López y Morcillo, 2007).

De esta manera, Internet dejó de ser un instrumento especializado de la comunidad científica para transformarse en una red de fácil uso, modificando las pautas de interacción social, convirtiéndose en el instrumento de comunicación más rápido en crecimiento. Transformaciones telemáticas que propician nuevas formas de enseñanza como la tele educación interactiva educación a distancia a través de la plataforma que provee la Internet-. De manera que surgen los navegadores del conocimiento. Es sin duda otra sociedad, la sociedad del conocimiento (Riveros y Mendoza, 2008).

Hoy en día, internet se ha convertido en la principal TIC - tecnologías de la información y la comunicación- que se mantiene en uso en los países desarrollados, por lo que se convierte metafóricamente en el oráculo digital de nuestro tiempo, ofreciéndonos una visión caleidoscópica de nuestra cultura (Aguiar y Cuesta, 2009). De esta manera, internet, permite romper ese monopolio del saber. Cualquier alumno puede acceder al website o espacio virtual no sólo de su profesor, sino también a una enorme variedad de recursos, sitios web, blogs, bases de datos, etc., relacionados con el curso que está estudiando (Area y Adell, 2009).

Cabe mencionar que Internet, en particular, y las TIC en términos generales, no son más que una herramienta para conseguir unos objetivos: mejorar la enseñanza y, por lo tanto, conseguir un mayor y mejor rendimiento académico del alumnado. Pero hay que tener claro que, como toda herramienta, hay que saber usarla o puede tener los efectos contrarios a los buscados (Bausela, 2009). 
Por lo tanto, Internet es una herramienta poderosísima a nuestro alcance, un lugar de encuentro virtual, un medio de comunicación y una fuente de informaciones diversas, pone a nuestro alcance como docentes una poderosa posibilidad, que es la de publicar nuevos contenidos y hacer partícipe al alumnado en su propio proceso de aprendizaje pasando de ser un alumno pasivo que recibe la información a un alumno activo que participa en la elaboración de la misma (Albarrán, 2010).

Dado que, en la educación actual, internet ofrece diferentes servicios que permiten ser utilizados tanto por el alumnado como por el profesor para llevar a cabo la recaudación de información para la materia y la impartición de las clases respectivamente. Eso es pues, mediante esta herramienta se establece una comunicación y al mismo tiempo el alumnado se está familiarizando cada vez más con las nuevas tecnologías. El uso de las mencionadas nuevas tecnologías, permite que el alumnado desarrolle competencias lingüísticas, gramaticales y comunicativas, así como a saber corregir sus propios errores y a expresarse de la mejor manera posible (Contreras, 2010).

Es justo decir que la Real Academia Española incluyo el vocablo "Internet" como un sustantivo en 2006 y la definió como una "red informática mundial, descentralizada, formada por la conexión directa entre computadoras u ordenadores mediante un protocolo especial de comunicación". Este protocolo o lenguaje común se denomina TCP/IP -"Transmisión Control Protocol/Internet Protocol"- el cual realiza un intercambio de información (Conesa, 2010). Cabe señalar que, Internet, permite romper ese estereotipo del saber. Cualquier estudiante puede acceder y rebasar de manera incalculable los conocimientos de su profesor y, por extensión, del resto del mundo. Así de esta forma los estudiantes tienen la posibilidad de encontrar una enorme variedad de información que puede enriquecer de manera significativa su proceso de aprendizaje (Andrade, 2011).

Lo cierto es que la tecnología ha cambiado fundamentalmente el proceso de educación de las personas. El conocimiento ya no está reservado a quienes tienen acceso a la información reposada en bibliotecas y facultades. En la actualidad, cada persona debe jugar un rol activo en su adquisición de conocimientos sin depender de los demás, de esta manera, internet tiene la habilidad de nivelar el campo de oportunidades para todos (Gómez y Macedo, 2011). 
Es así, como podemos señalar que el uso de internet de manera moderada se convierte en algo constructivo para el alumno. Si son muchas las veces en las que nos preocupamos por la escasa motivación de los estudiantes, ¿por qué no aprovechar los avances que nos proporciona las nuevas tecnologías? (Muñoz, Fragueiro y Ayuso, 2013).

Para finalizar, gracias al fácil acceso a la información, la educación ha podido avanzar mucho. En la actualidad se puede lograr el aprendizaje si se utiliza internet como fuente de información, sin embargo, se conoce poco cuáles son las estrategias de comprensión que los usuarios ponen en práctica cuando usan internet (Costa, Cuzzocrea y Nuzzaci, 2014).

\section{Materiales y Métodos}

De esta manera, el siguiente trabajo de investigación es no experimental de tipo descriptivo, el cual se llevó a cabo dentro de las aulas de la preparatoria 14 de la Universidad Autónoma de Nayarit (figura 1).

Figura 1. Alumnos consultando Internet en el centro de cómputo de la preparatoria 14.

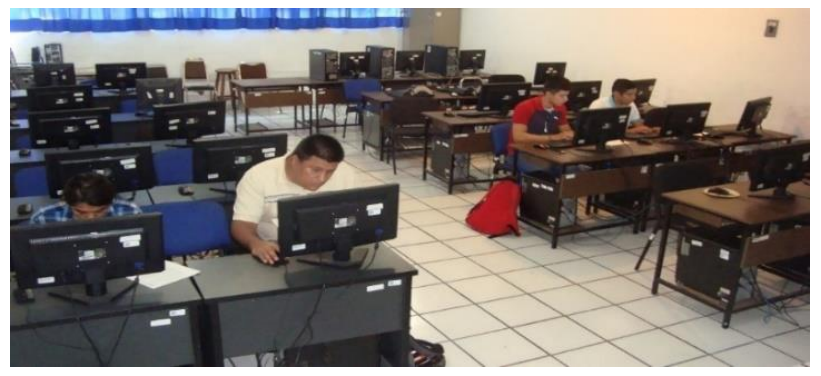

Fuente: elaboración propia.

Por lo tanto, considerando una población total de 383 alumnos inscritos para este ciclo escolar 2016 en la Unidad Académica Preparatoria 14. Se empleó la siguiente fórmula estadística de población finita y conocida (Murray y Larry, 2009):

$$
n=\frac{\mathrm{Z}_{\alpha}^{2} \cdot N \cdot p \cdot q}{i^{2}(N-1)+Z_{\alpha}^{2} \cdot p \cdot q}
$$


Basándose en un nivel de confianza $(Z)$ del 95\% y un error de estimación máximo aceptado (i) del 5\%, con el 50\% de población que tiene el atributo deseado (p), y con 50\% de la población que no tiene el atributo deseado (q), se obtuvo una muestra (n) de 192 alumnos.

Asimismo, el instrumento utilizado fue un cuestionario estructurado con 10 preguntas de tipo cerrada, el cual se aplicó a una muestra de 192 alumnos para los grados de primero, segundo y tercer año de la preparatoria 14, que hacen uso de esta herramienta tecnológica para desarrollar ciertas actividades académicas.

Cabe mencionar que para logar el objetivo de la investigación, es necesario estudiar las siguientes variables: 1.- determinar el acceso a internet desde el hogar de los estudiantes, 2.- el tipo de conexión a internet, 3.- como aprendió a utilizar internet, 4.- que tipo de navegador utiliza para navegar en internet, 5.- cuantas horas se conecta a internet, 6.- en qué lugar hace uso de internet, 7.- que motor de búsqueda emplea constantemente para buscar y/o consultar información en la red, 8.- qué tipo de información consulta en Internet, 9.- cita la información consultada en el internet, 10.- con qué frecuencia el profesor te pide hagas uso de las tecnologías.

De lo anterior, la información adquirida se procesó en el programa estadístico SPSS versión 19, obteniendo las pertinentes tablas de contingencia, para después presentar los datos con sus respectivas gráficas.

\section{Resultados}

\section{¿Tienes acceso a internet desde tu hogar?}

Al preguntar a los encuestados sobre si tiene acceso a internet desde su hogar, el 76\% respondió que sí y el 24\% respondió que no. Por consiguiente, el 39.1\% respondió que sí y el 13.5\% que no, respectivamente para primer año. Para segundo año tenemos que el 18.8\% respondió que sí y el $5.7 \%$ que no. Para tercer año el $18.2 \%$ respondió que sí y el $4.7 \%$ que no. La importancia de contar con internet en el hogar es prioritaria para el desarrollo de tareas y consulta de la información, mientras que el resto no cuenta con este tipo de herramientas en el hogar imposibilitando el acceso a este tipo de herramientas, como se observa en el Gráfico 1. 
Gráfico 1. ¿Tienes acceso a internet desde tu hogar?

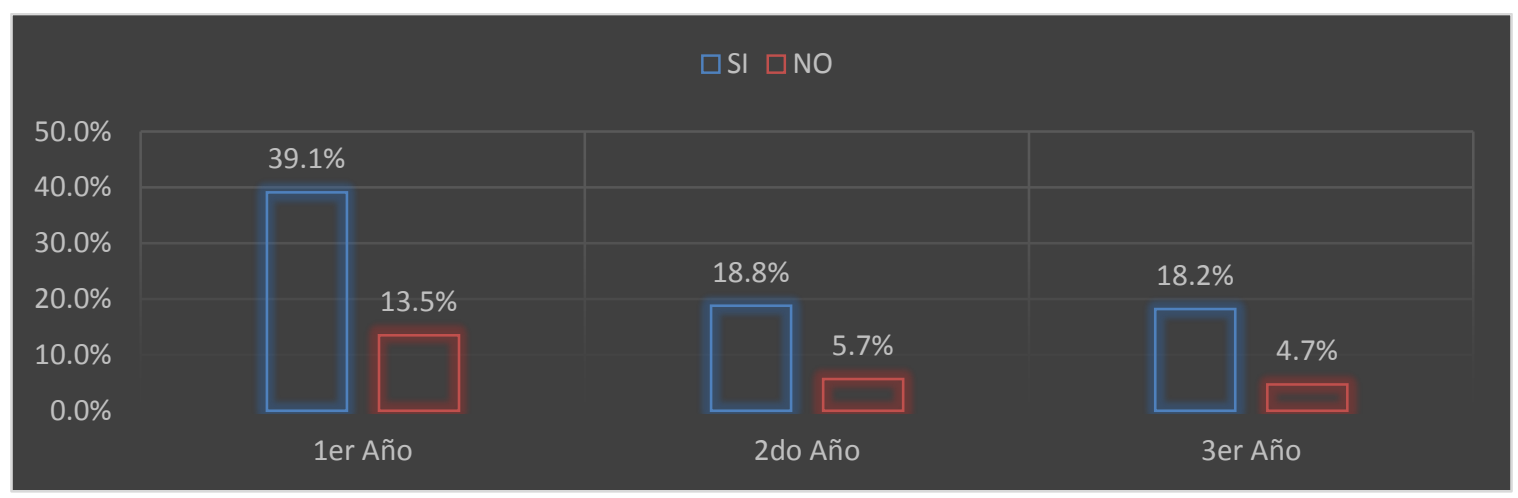

Fuente: elaboración propia.

\section{¿Qué tipo de conexión tienes en tu hogar?}

Con respecto a qué tipo de conexión tienes en tu hogar se presentan los siguientes resultados. Con un total del 1.6\% tiene conexión ADSL, el 15.1\% conexión telefónica, el $20.3 \%$ conexión cable, el $37.5 \%$ conexión inalámbrica, el $8.3 \%$ cuenta con otro tipo de conexión, y el 17.2\% no sabe qué tipo de conexión tiene. Se comprende que para el primer año tenemos que el $1 \%$ cuenta con conexión ADSL, el $7.3 \%$ con conexión telefónica, el 14.6\% con conexión cable, el 19.8\% con conexión inalámbrica, el 2.6\% otro tipo de conexión, el 7.3\% no tiene. Para segundo año, tenemos que el $0.5 \%$ cuenta con conexión ADSL, el 3.6\% conexión telefónica, el $1.6 \%$ conexión cable, el $10.4 \%$ conexión inalámbrica, el $2.6 \%$ otro tipo de conexión y el $5.7 \%$ no tiene.

Para tercer año, el $0 \%$ conexión ADSL, el 4.2\% conexión telefónica, 4.2\% conexión cable, el 7.3\% conexión inalámbrica, el 3.1\% cuenta con otro tipo de conexión y el $4.2 \%$ no cuenta con ningún tipo de conexión, así pues, los rubros con mayor porcentaje se presentan con la conexión cable y conexión inalámbrica, que es el tipo de conexión que prefieren las personas tener en su hogar, como se observa en el Gráfico 2. 
Gráfico 2. ¿Qué tipo de conexión tienes en tu hogar?

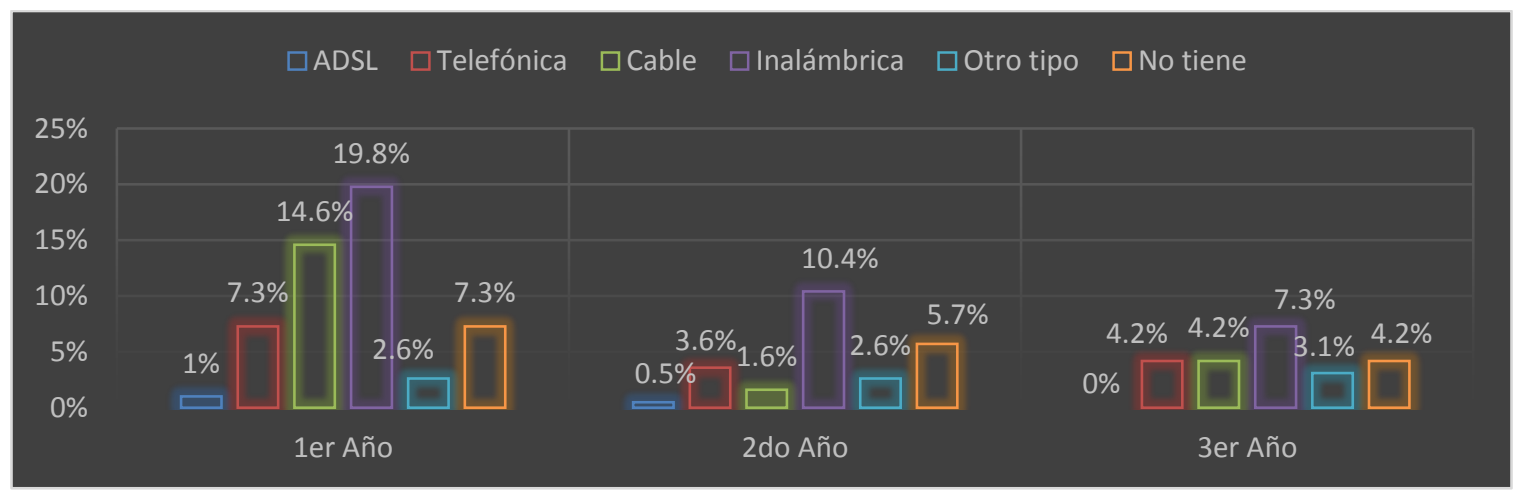

Fuente: elaboración propia.

\section{¿Cómo aprendió a usar Internet?}

Así pues, para primer año el 35.4\% aprendió a usar internet por el mismo, el $5.2 \%$ por cursos de informática, el $10.9 \%$ por amigos o familiares, el $1 \%$ por otros medios. Para segundo año, el $17.7 \%$ por el mismo, el $1.6 \%$ cursos de informática, el 3.6\% por amigos o familiares y el $1.6 \%$ por otros medios. Asimismo, para tercer año el $9.9 \%$ aprendió por el mismo, el $7.8 \%$ por cursos de informática, el $4.2 \%$ por amigos o familiares y el $1 \%$ por otros medios, como se observa en el Gráfico 3.

Gráfico 3. ¿Cómo aprendió a usar Internet?

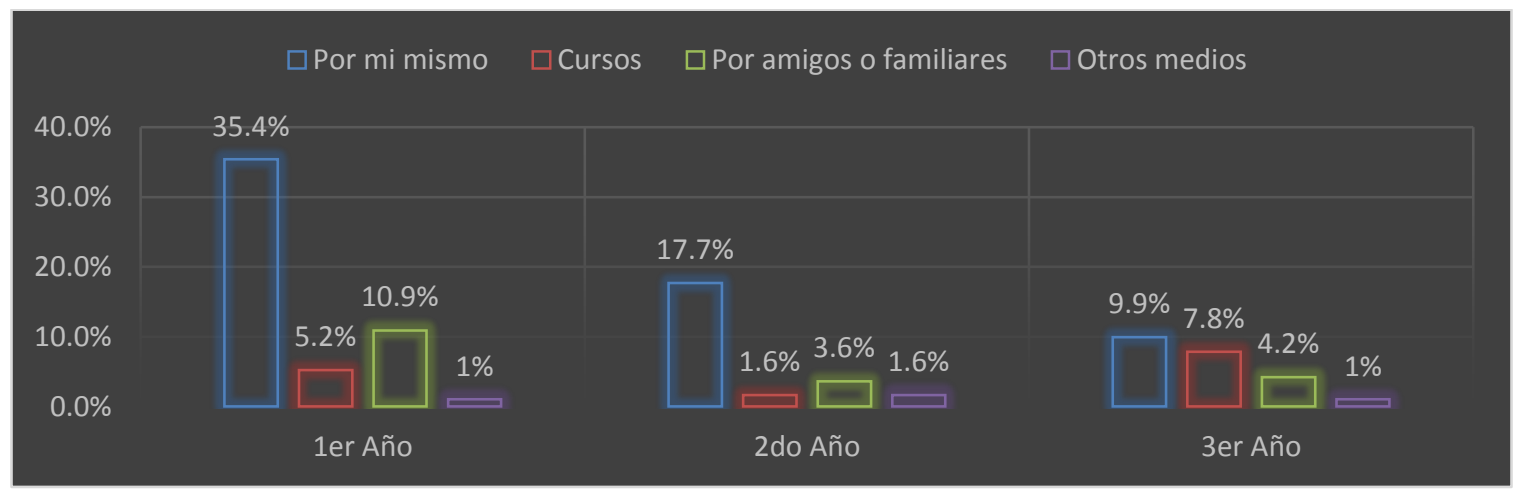

Fuente: elaboración propia. 


\section{¿Qué tipo de navegador utilizas para hacer uso de Internet?}

Esto quiere decir que del total de los alumnos encuestados $19.3 \%$ utiliza el navegador internet Explorer, el $72.4 \%$ el Google Chrome, el 3.1\% Firefox, el 3.6\% Safari, y el 1.6\% utiliza otro navegador. Lo que quiere decir que el 9.9\% utiliza Internet Explorer, el 39.1\% Google Chrome, el 1\% Firefox, el $2.6 \%$ el Safari, correspondiente al primer año. Para el segundo año, el $4.7 \%$ utiliza Internet Explorer, el 17.7\% Google Chrome, el 1\% Firefox, el 1\% utiliza otro navegador. Para tercer año, el 4.7\% utiliza Internet Explorer, el 15.6\% Google Chrome, el 1\% Firefox, el 1\% Safari, el $0.5 \%$ utiliza otro navegador. Cabe señalar que los alumnos prefieren usar el navegador Google Chrome, por ser un navegador confiable y eficaz dentro de la consulta de la información, manteniendo una velocidad aceptable para navegar, y hacer uso de los recursos que ofrece la red, como se observa en el Gráfico 4.

Gráfico 4. ¿Qué tipo de navegador utilizas para hacer uso de Internet?

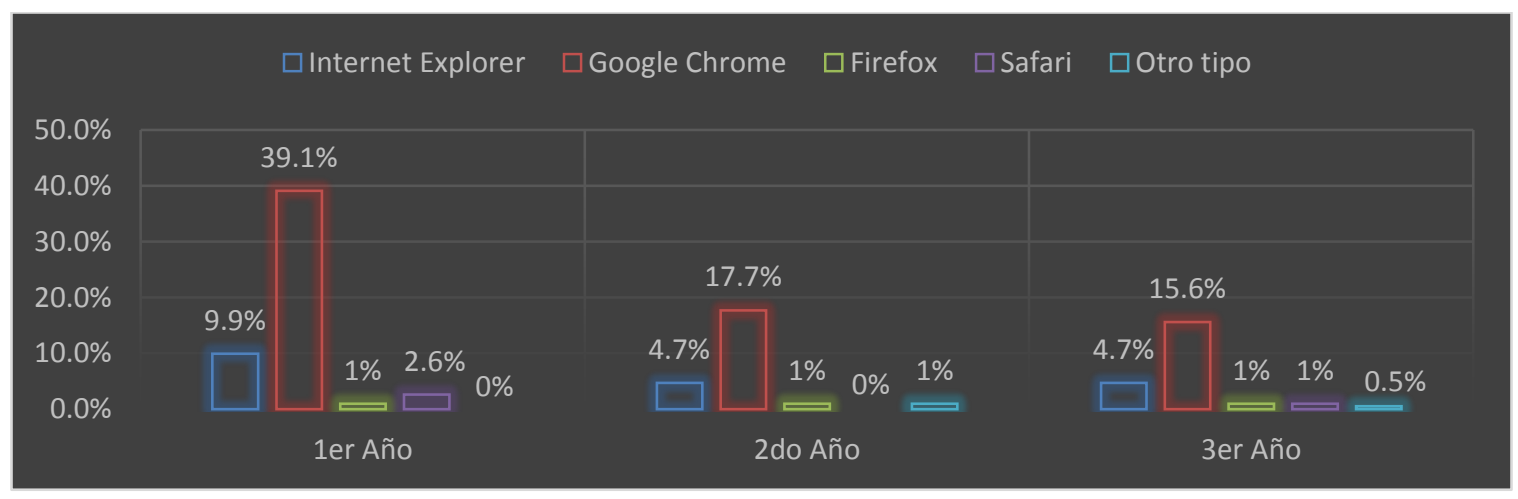

Fuente: elaboración propia.

\section{¿Cuántas horas se conecta a Internet en la semana?}

Con respecto a cuantas horas se conecta el alumno a internet en la semana el resultado total es, menos de dos horas el 24\%, tres horas el 20.3\%, seis horas el 19.8\%, más de diez horas el 20.8\%, no sabe el $15.1 \%$. De lo anterior tenemos que para primer año el $13 \%$ se conecta menos de dos horas, el $10.9 \%$ tres horas, el $11.5 \%$ seis horas, el 9.4\% más de diez horas, el $7.8 \%$ no sabe. Para segundo año, el 5.7\% menos de dos horas, $4.7 \%$ tres horas, el 3.6\% seis horas, $6.8 \%$ más de diez horas, el 3.6\% no sabe. Para el tercer año, el 5.2\% menos de dos horas, el $4.7 \%$ tres horas, el 4.7\% seis horas, el $4.7 \%$ más de diez horas, y el 3.6\% no sabe, podemos determinar, que más de 
diez horas el alumno hace uso del internet en la semana, en la consulta de la información y/o en actividades lúdicas ajenas a la educación, como se observa en el Gráfico 5.

Gráfico 5. ¿Cuántas horas se conecta a Internet en la semana?

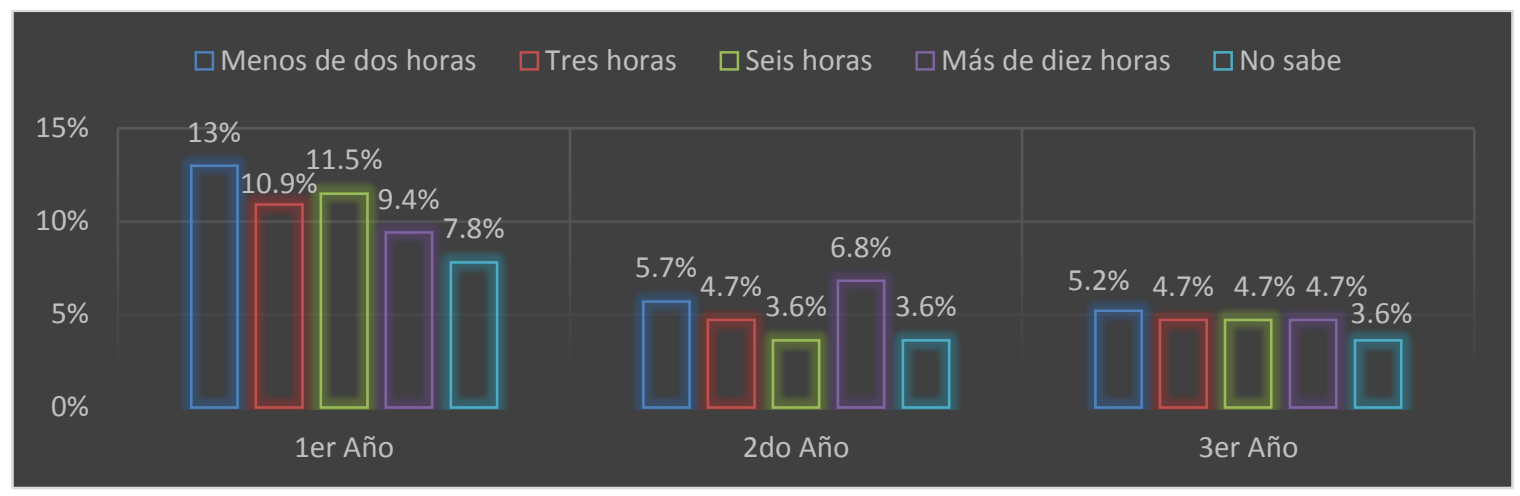

Fuente: elaboración propia.

\section{¿En qué lugar haces uso del internet para realizar tus consultas y/o tareas?}

Por tanto, para los alumnos que hicieron uso de internet desde casa, para el primer año $44.8 \%$, para segundo año el $17.7 \%$, y para el tercer año $18.2 \%$, en la escuela, para primer año $0.5 \%$, el $0.5 \%$ para segundo año y $0.5 \%$ para tercer año, en biblioteca para el primer año $0.5 \%$, para segundo año el $0.5 \%$. y para tercer año no presenta resultados, para los alumnos que hacen uso en el cibercafé tenemos que para primer año es de $4.2 \%$, para segundo año $3.6 \%$ y $2.6 \%$ para tercer año, para los alumnos que hacen uso de internet desde el trabajo, para primer año $2.1 \%$, segundo año $2.1 \%$, y tercer año $0.5 \%$, y para los que hacen uso de internet en otro lugar tenemos que para primer año es el $0.5 \%$, segundo año no presenta resultados, y tercer año el $1 \%$, sin embargo se observa la necesidad de algunos alumnos que recurren al uso del internet desde otro lugar para desarrollar actividades académicas como lúdicas, como se observa en el Gráfico 6. 
Gráfico 6. ¿En qué lugar haces uso del internet para realizar tus consultas y/o tareas?

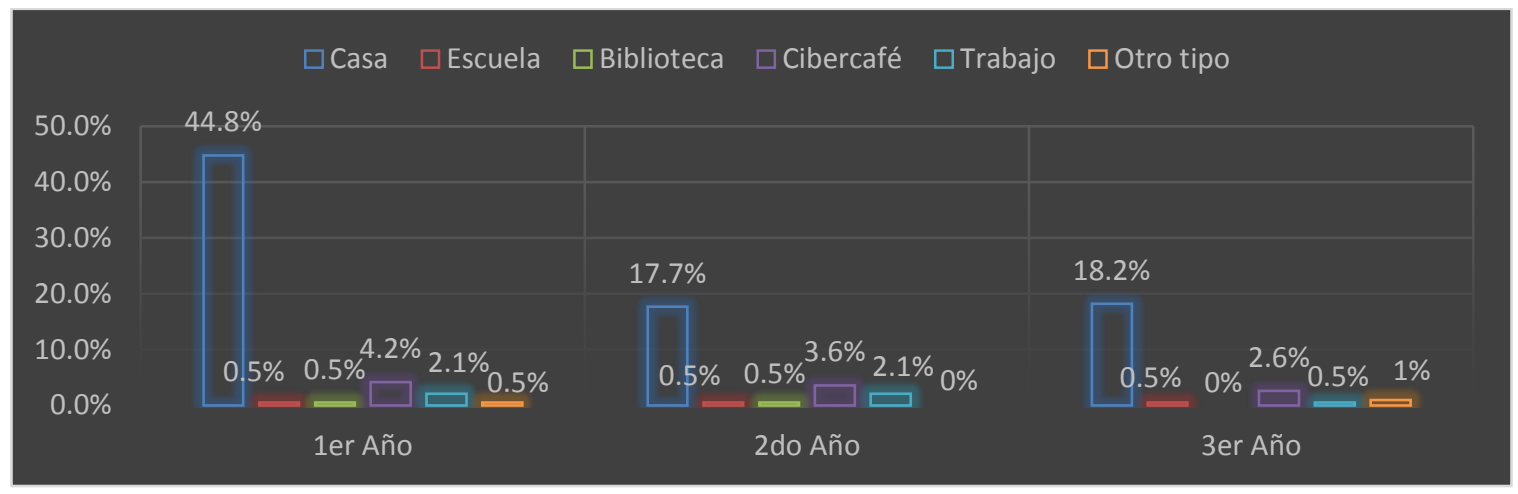

Fuente: elaboración propia.

¿Cuál de estos motores de búsqueda empleas constantemente para buscar y/o consultar información en la red?

Los resultados presentados tenemos en un total que el $91.7 \%$ utiliza el Google, el 1\% Bing, el 4.7\% Windows Live, el 1\% Yahoo, y el 1.6\% utiliza otro motor de búsqueda.

Por tanto, para primer año los resultados son, el 46.4\% utiliza Google, el 1\% Bing, el 2.6\% Windows Live, el 1\% Yahoo, $1.6 \%$ otro tipo de buscador. Para segundo año, el $22.4 \%$ Google, el 0\% Bing, el 2.1\% Windows Live, 0\% Yahoo, 0\% otro tipo de buscador. Y para tercer año, el 22.9\% Google, respectivamente para Bing, Windows Live, Yahoo, y otro tipo de buscador no presentan resultados, como se observa en el Gráfico 7. 
Gráfico 7. ¿Cuál de estos motores de búsqueda empleas constantemente para buscar y/o consultar información en la red?

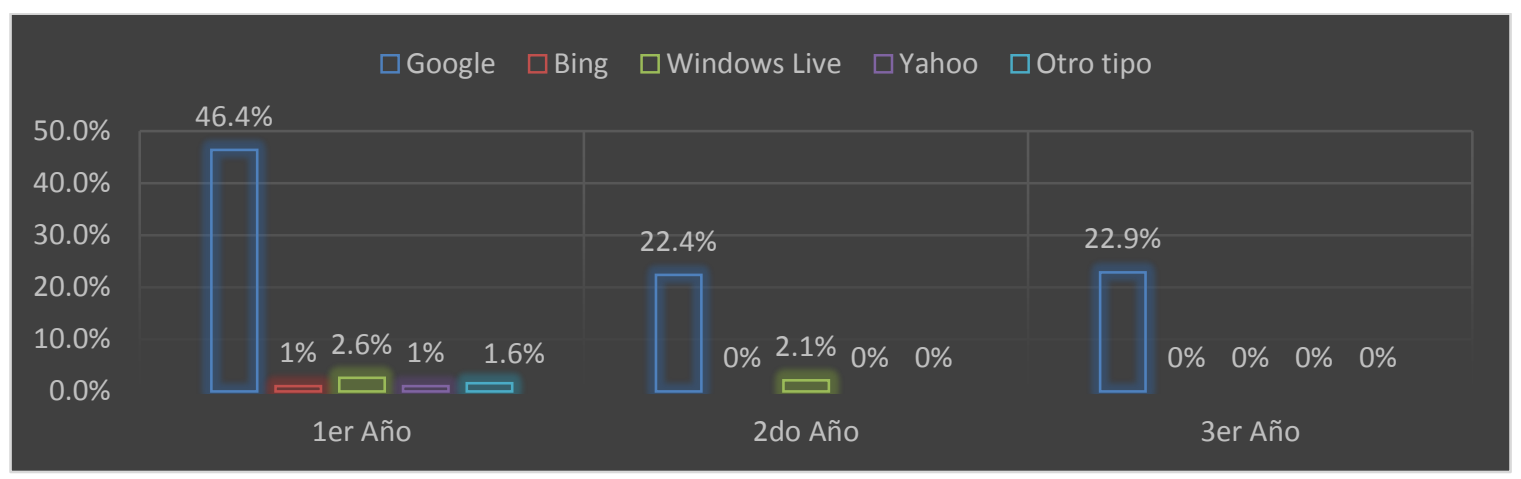

Fuente: elaboración propia.

\section{¿Qué tipo de información consultas en Internet?}

Con respecto a que información se consulta en internet tenemos el siguiente resultado total, el $64.6 \%$ consulta información de tipo educativa, el 9.9\% científica, $10.9 \%$ social, el 1\% negocios, el 1\% política, el 3.6\% deportiva, el 1.6\% religiosa, el 7.3\% otro tipo de información. Para primer año, el $32.8 \%$ consulta información educativa, el $5.7 \%$ científica, $5.2 \%$ social, el $1 \%$ negocios, el $1 \%$ política, el $2.6 \%$ deportiva, no consulta información religiosa, el $4.2 \%$ consulta otro tipo de información. El segundo año, presenta que el 15.1\% educación, 3.1\% científica, el 3.1\% social, no consulta, no consulta, el $0.5 \%$ deportiva, el $1 \%$ religiosa, el $1.6 \%$ otro tipo de información. Para tercer año, el $16.7 \%$ educativa, $1 \%$ científica, el $2.6 \%$ social, no consulta, no consulta, el $0.5 \%$ deportiva, el $0.5 \%$ religiosa, y el $1.6 \%$ otro tipo, como se observa en el Gráfico 8.

Gráfico 8. ¿Qué tipo de información consultas en Internet?

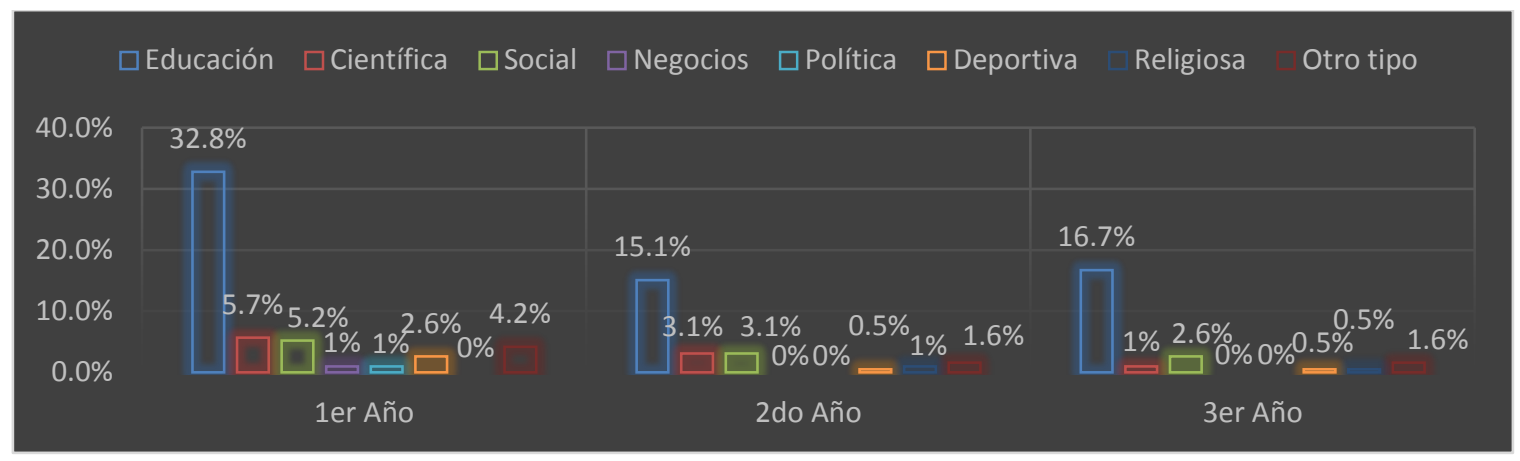

Fuente: elaboración propia. 


\section{¿Cuándo consultas y/o haces uso de la información que está disponible en Internet citas la fuente?}

Del total de alumnos encuestados el $27.6 \%$ respondió que nunca, el 54.2\% ocasionalmente, el $14.6 \%$ constantemente, el $3.6 \%$ muy constante. De lo anterior tenemos que, para primer año, el $12.5 \%$ respondió que nunca, el $33.9 \%$ ocasionalmente, el $5.7 \%$ constantemente, $0.5 \%$ muy constante. El segundo año, el 7.8\% respondió que nunca, el 9.4\% ocasionalmente, el 5.7\% constantemente, el 1.6\% muy constante. Para tercer año, con el $7.3 \%$ que nunca, $10.9 \%$ ocasionalmente, $3.1 \%$ constantemente, el $1.6 \%$ muy constante, se observa que, dentro del panorama, la mayoría de los estudiantes cita las fuentes de información ocasionalmente, lo que demuestra que la mayoría de los alumnos no se le exige citar la fuente consultada y dar crédito a los autores por el trabajo intelectual presentado en la red, como se observa en el gráfico 9.

Gráfico 9. ¿Cuándo consultas y/o haces uso de la información que está disponible en Internet citas la fuente?

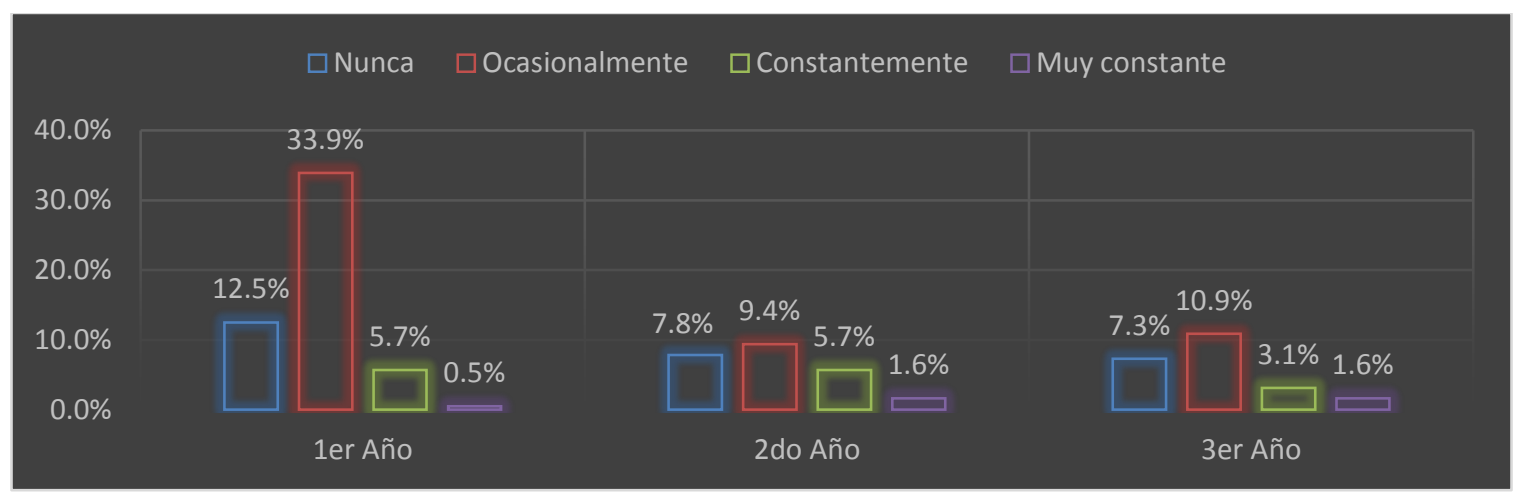

Fuente: elaboración propia.

¿Con qué frecuencia el profesor te pide hagas uso de las tecnologías -internet, motores de búsqueda, programas, tutoriales, email, foros, etc.-?

Los resultados obtenidos fueron los siguientes, con un total el 3.6\% respondió que nunca, el $35.4 \%$ respondió ocasionalmente, el $43.8 \%$ constantemente, y el 17.2\% muy constantemente. Los resultados para primer año, el $3.1 \%$ que nunca, el $21.9 \%$ ocasionalmente, el $21.9 \%$ constantemente, el 5.7\% muy constante. Para segundo año, el $0.5 \%$ nunca, el $9.9 \%$ ocasionalmente, el $8.9 \%$ constantemente, el 5.2\% muy constante. Y para tercer año, el 0\% 
respondió que nunca, el $3.6 \%$ ocasionalmente, el 13\% constantemente, $6.3 \%$ muy constante, posteriormente la falta de uso y enseñanza de estas tecnologías, ha limitado tanto al docente y alumno en integrar este tipo de herramientas en sus clases cotidianas, como se observa en el Gráfico

Gráfico 10. ¿Con qué frecuencia el profesor te pide hagas uso de las tecnologías -internet, motores de búsqueda, programas, tutoriales, email, foros, etc.-?

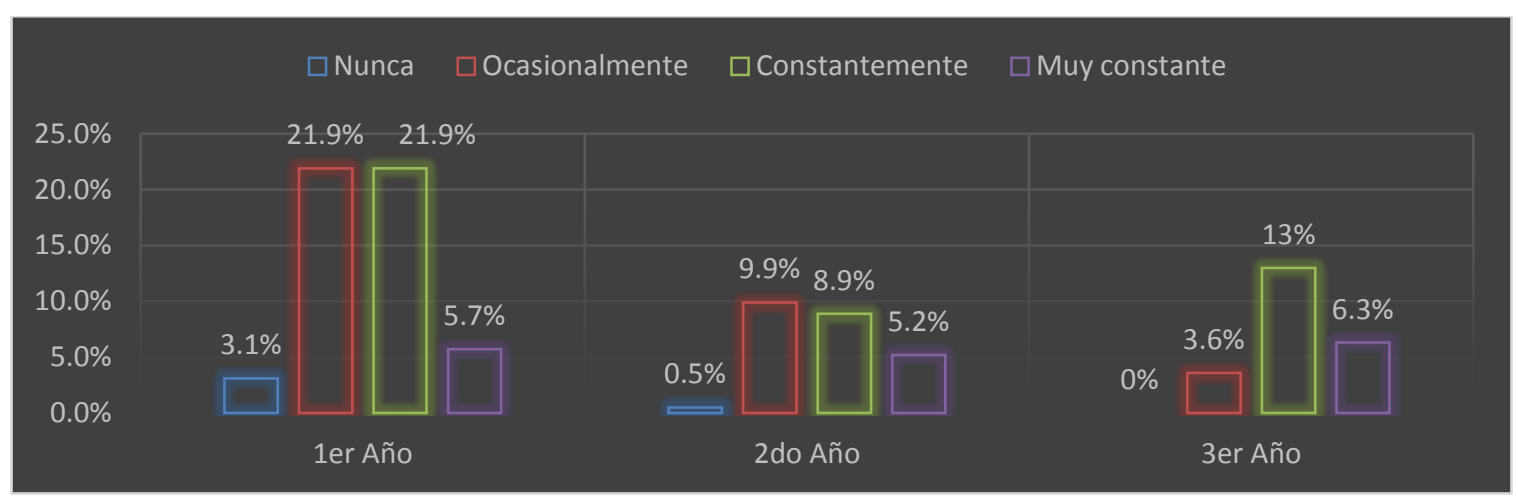

Fuente: elaboración propia.

\section{Conclusión}

Por lo tanto, se puede referir a Internet como la TIC más empleada e importante dentro del entorno educativo de la preparatoria 14. Siendo esta una herramienta didáctica tan significativa dentro de las actividades académicas de los estudiantes del sistema medio superior.

Asimismo, Araiza (2005) afirma que el uso de internet dentro de la educación tiene múltiples opciones; como son, el que los alumnos vivan la globalización realizando un intercambio de información con cualquier institución o persona alrededor del mundo, el elaborar investigaciones académicas y establecer un nuevo sistema virtual de comunicación ya sea sincrónico o asincrónico, entre sus compañeros y maestros. Cabe señalar que es una forma de trabajo que, al no requerir la presencia física, obvia las dificultades que presentan el tiempo y el espacio.

De lo anterior, se puede observar la trascendencia que genera en los estudiantes en la consulta de la misma información, utilizando gran variedad de motores de búsqueda, así como la diversidad de lugares para hacer uso del Internet. Asimismo, da la oportunidad de seguir con una educación basada en Tecnologías de Información y Comunicación. 


\section{Bibliografía}

Adell, J. (2004). Internet en educación. Comunicación y Pedagogía, núm. 200: 25-28.

Aguiar Perera, M. V.; Cuesta Suárez, H. (2009). Importancia de trabajar las TIC en educación infantil a través de métodos como la Webquest. Pixel-Bit. Revista de Medios y Educación, núm. 34, enero, 81-94.

Albarrán Vergel, M. S. (2010). La Webquest como recurso didáctico. Revista Autodidacta, 1(2): 96-109.

Andrade Ramírez, G. (2011). Aspectos negativos del uso del internet en la educación superior. Cuadernos de Educación y Desarrollo, 3(27): 2-10.

Araiza Guerra, J. (2005). El uso de internet como herramienta en las clases presenciales. (Tesis de maestría en educación superior). Nuevo León: Universidad Autónoma de Nuevo León.

Area, M. y Adell, J. (2009). eLearning: Enseñar y aprender en espacios virtuales. En J. De Pablos (Coord.): Tecnología Educativa. La formación del profesorado en la era de internet. Aljibe, Málaga, 391-424.

Bausela Herreras, E. (2009). La universidad en la sociedad red. Usos de internet en educación superior. Revista de la Educación Superior, XXXVIII (3): 181-184.

Conesa Fuentes, M. C. (2010). Evaluación de la calidad de los sitios web con información sanitaria en castellano (Tesis Doctoral). España: Universidad de Murcia. Recuperado en: http://www.tesisenred.net/bitstream/handle/10803/10916/ConesaFuentes.pdf?sequence=1 (27/08/2016).

Contreras Arroyo, B. (2010). Internet en la Educación. Innovación y Experiencias Educativas, núm. 29, abril, 1-9.

Costa, S.; Cuzzocrea, F. y Nuzzaci, A. (2014). Usos de internet en contextos educativos informales: Implicaciones para la educación formal. Comunicar, XXII (43): 163-171.

Dari, N. L. (2004). Entre riesgos y promesas: Educación digital. Revista electrónica de investigación educativa, 6(2): 2-6. 
Gómez Gallardo, L. M. y Macedo Buleje, J. C. (2011). Importancia de los programas virtuales en la educación superior Peruana. Investigación Educativa, 15(27): 113-126.

López García, M. y Morcillo Ortega, J. G. (2007). Las Tic en la enseñanza de la biología en la educación secundaria: los laboratorios virtuales. Revista Electrónica de Enseñanza de las Ciencias, 6(3): 562-576.

Muñoz Prieto, M. M.; Fragueiro Barreiro, M. S.; Ayuso Manso M. J. (2013). La importancia de las redes sociales en el ámbito educativo. Escuela Abierta, núm. 16: 91-104.

Murray R., S. y Larry J., S. (2009). Estadística Schawn. 4ta. Edición, Ed. Mc Graw-Hill. México, D.F., 577 pp.

Pérez Gutiérrez, A. y Florido Bacallao, R. (2003). Internet: un recurso educativo. Eticanet, 1(2): $1-12$.

Riveros, V. S. y Mendoza, M. I. (2008). Consideraciones teóricas del uso de la internet en educación. Omnia, 14(1): 27-46. 